\title{
Vascular endothelial growth factor suppresses dendritic cells function of human prostate cancer
}

This article was published in the following Dove Press journal:

OncoTargets and Therapy

\author{
Wen-kun Bai \\ Wei Zhang \\ Bing $\mathrm{Hu}$
}

Department of Ultrasound in Medicine, Shanghai Jiao Tong University Affiliated 6th People's

Hospital, Shanghai Institute of Ultrasound in Medicine, Shanghai, China
Correspondence: Bing Hu

Department of Ultrasound in Medicine, Shanghai Jiao Tong University Affiliated 6th People's Hospital, Shanghai Institute of Ultrasound in Medicine, Yishan Road 600, Shanghai China 200233

Tel +86 2I 64369 I8I, ext 8877

Fax +86 21 54488254

Email binghu_stan@163.com
Purpose: Prostate cancer (PCa) patients often have dendritic cell (DC) function defects, but the mechanism is not clear. The aim of this study was to detect the effect of vascular endothelial growth factor (VEGF) in mature DCs.

Patients and methods: In this study, we chose 30 PCa patients, 10 prostatic intraepithelial neoplasia (PIN) patients and 30 benign prostatic hyperplasia (BPH) patients, and compared the composition of peripheral blood $\mathrm{T}$ cells, the composition and function of local dendritic cells in prostate tissue, and the density of local VEGF.

Results: The results showed that the numbers of total DCs, mature and functional DCs, and $\mathrm{CD}^{+} \mathrm{T}$ cells were inhibited in $\mathrm{PCa}$, and the inhibitory effect was enhanced with increased malignancy. In addition, the infiltration density of VEGF-positive cells was increased in PCa, and this increase was associated with an increased malignant degree of PCa. The inhibition of tumor immunity in patients with PCa is achieved by inhibiting the function of dendritic cells.

Conclusion: VEGF plays an important role in the inhibition of the maturation and function of dendritic cells, and this inhibition is gradually increased with an increasing malignant degree of PCa.

Keywords: prostate cancer, vascular endothelial growth factor, dendritic cell, immunology, $\mathrm{T}$ cell

\section{Introduction}

Prostate cancer ( $\mathrm{PCa}$ ) is the most frequently diagnosed cancer and the second leading cause of cancer death in males in the United States, accounting for $27 \%(233,000)$ of total new cancer cases and $10 \%(29,480)$ of total cancer deaths in males in $2014 .{ }^{1}$ Beyond traditional surgery, radiotherapy and hormone deprivation methods, many studies focus on dendritic cell-based immunotherapy since it is a novel treatment for advanced $\mathrm{PCa}{ }^{2}$ Dendritic cells (DCs) are the most important antigen-presenting cell population for regulating innate and adaptive immune responses. They are required to convert naïve resting $\mathrm{T}$ cells into potent tumor-reactive cytotoxic $\mathrm{CD} 8^{+}$and helper $\mathrm{CD}^{+} \mathrm{T}$ cells. The activation of DCs can effectively stimulate B lymphocytes to produce a specific antibody - $\mathrm{T}$ cell transformation into cytotoxic $\mathrm{T}$ cells produces an effective immune response, ${ }^{3}$ and plays an important role in tumor immunity. The immune response of DCs is mainly mediated by $\mathrm{CD}^{+}$and $\mathrm{CD} 4^{+} \mathrm{T}$ cells. The secretion of various cytokines produced by $\mathrm{CD} 4^{+}$cells, and a part of them influence $\mathrm{CD} 8^{+}$ cells differentiation development to $\mathrm{CD}^{+}$cytotoxic $\mathrm{T}$ cells, and by $\mathrm{CD} 8^{+}$cytotoxic $\mathrm{T}$ cells, leads to the activation of the perforin/pathway and Fas/FasL pathways and the killing of target cells. DCs also play a major role in the immune memory, which can stimulate the formation of $\mathrm{T}$ cell differentiation and expansion to form antigenspecific killer T cells. ${ }^{4}$ 
Early theories did not consider the prostate to be an organ monitored by the body's immune system, ${ }^{5}$ but nearly 7 years of research has overthrown this viewpoint, suggesting that the treatment of PCa may potentially work by activating the immune system. ${ }^{6}$ Therefore, increasing attention to the clinical treatment of $\mathrm{PCa}$, especially in late androgenindependent or distant metastasis, focuses on activating the human immune system to treat PCa. Sipuleucel-T was approved by the United States Food and Drug Administration for PCa therapy in 2010. ${ }^{7}$

Previous studies have demonstrated that the quantity and activity of DCs in PCa patients are significantly lower than in healthy controls,${ }^{8}$ and in vitro experiments verified that PCa cells induce apoptosis, as well as suppress the regeneration and differentiation of DCs. ${ }^{9}$ Studies also showed that the numbers of DCs are reduced in $\mathrm{PCa}$ tissues compared to prostatic hyperplasia (BPH) tissues, and the levels of infiltrating DCs are further decreased with the advancement of atypia. ${ }^{10}$ Current immunotherapies are confined to relieve the symptoms in advanced PCa patients and cannot cure the disease, and the immunosuppressive tumor microenvironment may limit their effectiveness. ${ }^{11}$ Therefore, it is necessary to determine the mechanism of immune inhibition to overcome the immune restriction and improve the efficacy of immunotherapy.

Vascular endothelial growth factor (VEGF), which induces neoangiogenesis and the blockade of angiogenesis, plays an important role in the development and metastasis of solid tumors, and targeting VEGF has become a useful approach in cancer therapy. ${ }^{12}$ The inhibition of VEGF in a mouse model leads to increased antigen uptake and the migration of tumor-associated DCs. ${ }^{13}$ In the human tongue carcinoma, cancer cells could be inhibited in proliferation and be increased in apoptosis by knocking down the expression of VEGF, and maturation of DCs also could be improved. ${ }^{14}$ VEGF also influences the effect of DCs on therapy in the model of colon cancer-induced malignant ascites. ${ }^{15}$ VEGF expression in the peripheral blood and neoplasm nest from patients with oral squamous cell carcinoma (OSCC) was positively correlated with the course of the disease, while an inverse correlation between VEGF expression and DCs was identified in the peripheral blood. ${ }^{14}$ Some clinical research has been carried out that blocks VEGF to improve the antitumor responses in treating PCa patients. ${ }^{16} \mathrm{PCa}$ is divided into three categories, which fit the following standard definitions: low-risk PCa (biopsy Gleason grade $<7$ or prostate-specific antigen (PSA) $<10 \mathrm{ng} / \mathrm{mL}$ ), intermediate-risk $\mathrm{PCa}$ (biopsy Gleason score 7 or PSA $10-20 \mathrm{ng} / \mathrm{mL}$ ), and high-risk PCa (biopsy Gleason score $>7$ or PSA $>20 \mathrm{ng} / \mathrm{mL}$ ). ${ }^{17-20}$ Determining the status of DC and VEGF expression in different degrees of $\mathrm{PCa}$ patients is needed.

In this study, we compared the composition and functional status of DCs in prostate tissues of patients with $\mathrm{BPH}$, prostatic intraepithelial neoplasia (PIN) and $\mathrm{PCa}$ and compared the expression of VEGF in these prostate tissues. We also compared the changes of T-lymphocyte $\mathrm{CD} 4^{+} \mathrm{T}$ cells, $\mathrm{CD} 8^{+}$ $\mathrm{T}$ cells, and the $\mathrm{CD}^{+} / \mathrm{CD}^{+}$ratio in the $\mathrm{BPH}, \mathrm{PIN}$ and $\mathrm{PCa}$ groups. We examined the DCs in the PCa microenvironment, the $\mathrm{T}$ lymphocytes in peripheral blood, and the association of VEGF expression with Gleason scores and PSA levels in PCa patients. In addition, we studied the correlation between S100 expression, VEGF and CD208 in PCa patients.

\section{Materials and methods}

This study obtained permission from the ethics committee of the Shanghai Jiao Tong University Affiliated 6th People's Hospital and the Shanghai Institute of Ultrasound in Medicine, and written informed consent was obtained from each patient. Mice blood was used in this research and permission was also obtained from the ethics committee of the Shanghai Jiao Tong University Affiliated 6th People's Hospital and the Shanghai Institute of Ultrasound in Medicine. All experiments were performed following relevant Shanghai Jiao Tong University Affiliated 6th People's Hospital and national guidelines and regulations.

\section{Patients}

An ultrasound-guided 10- to 12-core transrectal systematic prostate biopsy was obtained from all patients based on the European Association of Urology (EAU) guideline suggestions. The patients had no history of blood transfusions, no acute or chronic infectious diseases, and they did not use any immunomodulatory drugs or treatment, receive radiation therapy, or have autoimmune diseases. The peripheral blood levels of PSA, $\mathrm{CD}^{+} \mathrm{T}$ cells, and $\mathrm{CD} 8^{+} \mathrm{T}$ cells, as well as the $\mathrm{CD}^{+} / \mathrm{CD} 8^{+}$ratio, were measured before the procedure.

\section{Groups}

The patients were divided into three groups according to their pathological results: BPH, PIN and PCa. The PCa patients were divided into three groups according to their PSA levels: group A, PSA < $10 \mathrm{ng} / \mathrm{mL}$; group B, PSA 10-20 ng/mL; and group C, PSA $>20 \mathrm{ng} / \mathrm{mL}$.

PCa patients were also stratified according to Gleason score: group D, Gleason grade $<7$; group E, Gleason grade 7; and group F, Gleason grade $>7$. 


\section{Phenotypic analysis of $\mathrm{CD}^{+}, \mathrm{CD}^{+}$, and CD4/CD8 by flow cytometry}

Fresh peripheral venous blood was collected from all patients 1 day before biopsy. Anticoagulant $(100 \mu \mathrm{L})$ was added to each of the two test tubes containing either $20 \mu \mathrm{L} \mathrm{CD} 4-$ FITC (BD, Franklin Lakes, NJ, USA) or $20 \mu \mathrm{L}$ CD8-PE (BD) and mixed evenly. Additionally, a test tube with blood from Control mice and IgG1-FITC (BD) or IgG1-PE (BD) monoclonal antibodies $(20 \mu \mathrm{L})$ was also mixed. All test tubes were incubated at room temperature after mixing for $20 \mathrm{~min}$; then, $2 \mathrm{~mL}$ of red cell lysate (BD Biosciences, San Jose, CA, USA) was added. After incubation at room temperature for $10 \mathrm{~min}$, samples were centrifuged at 12,000 rpm for $5 \mathrm{~min}$, the supernatant was discarded, and $2 \mathrm{~mL}$ of phosphate buffer $\mathrm{pH} 7.4$ (0.05\% PBS) was added. The samples were mixed well and washed 1-2 times. Cells were resuspended in $500 \mu \mathrm{L}$ of PBS, and immediately tested by flow cytometry. We gated for small lymphocytes (R1 gate) based on the forward scatter and side scatter of the cells. Mouse IgG1-FITC and IgG1-PE monoclonal antibodies were used as isotypenegative controls.

The data were acquired using a FACScan flow cytometer (BD) and analyzed with CellQuest software (BD).

\section{PSA detection}

Fasting venous blood ( $4 \mathrm{~mL})$, which had been placed in sterile centrifuge tubes, was centrifuged at 3,000 rpm for $15 \mathrm{~min}$, and collected sera were placed in a sterile tube. PSA detection was done using a Swiss Roche E601 automatic electrochemical luminescence. Test results were automatically determined based on the standard curve.

\section{Immunohistochemistry}

The composition and functional status of DCs and the expression of VEGF (Santa Cruz Biotechnology, Inc., Dallas, TX, USA) in prostate tissues of patients were detected by immunohistochemistry. S100 (Santa Cruz Biotechnology, Inc.) is a specific marker of DCs, and CD208 (Santa Cruz Biotechnology, Inc.) is a specific marker for mature and functional DCs. ${ }^{21-23}$ Routine formalin-fixed paraffin-embedded 3-4- $\mu$ m-thick sections from each patient were attached to silanized slides, sequentially deparaffinized and rehydrated. Access to tissue antigen sites for antibody attachment was enhanced by microwaving slides, which were treated with citrate buffer for $20 \mathrm{~min}$. The primary antibody was diluted 1:100 in Tris buffered saline (TBS)/1\% BSA/1\% human serum and incubated for $30 \mathrm{~min}$. The EnVision technique and development with the chromogen 3,3'-diaminobenzidine tetrachloride was used for visualization. Sections were lightly counterstained with hematoxylin. ${ }^{24}$ All slides were examined by two trained pathologists. Negative staining slices also were completed.

Brown granular staining in the cytoplasm or nucleus was defined as S100- and CD208-positive results. VEGF-positive cells showed brown granular staining in the cytoplasm.

Measurements of DC and VEGF-positive cell infiltration density are expressed as the mean \pm standard deviation. The saturation density of DCs/VEGF-positive cells $=10 \times$ the high power field of view of positive cells $/ 2.5 \mathrm{~mm}^{2}$.

\section{Statistical analysis}

SPSS16.0 statistical software was used for statistical analyses. The data were subjected to single factor variance analysis. Further pairwise comparisons were conducted using the Student-Newman-Keuls test, and the correlation between VEGF and DCs was analyzed by correlation analysis. $P$-values $<0.05$ were considered statistically significant.

\section{Results}

\section{Age and PSA levels of the three groups (Table I)}

A total of $30 \mathrm{BPH}$ patients, $10 \mathrm{PIN}$ patients and $30 \mathrm{PCa}$ patients were included in this study.

The PSA levels in patients with PCa and PIN were significantly different from those with $\mathrm{BPH}(P=0.010)$. There was no significant difference in PSA levels between BPH and PIN patients or PCa and PIN patients $(P>0.05)$. No significant differences in age were seen between the three groups $(P>0.05)$.

\section{Changes of peripheral blood CD4, CD8 and CD4/CD8 levels in three groups (Table 2)}

The expression level of CD4 in each group reduced with the malignant degree of disease, and there was a significant difference in CD4 levels between BPH and PCa patients

Table I Age and PSA levels in patients with different pathological types

\begin{tabular}{lll}
\hline Group & Age (year) & PSA (ng/mL) \\
\hline BPH & $67.47 \pm 10.39$ & $11.67 \pm 12.70$ \\
PIN & $69.50 \pm 11.13$ & $22.42 \pm 12.32$ \\
PCa & $67.97 \pm 11.91$ & $25.07 \pm 26.07^{*}$ \\
\hline
\end{tabular}

Notes: $* P=0.010$ vs BPH. Data are presented as mean \pm standard deviation.

Abbreviations: PSA, prostate-specific antigen; BPH, benign prostatic hyperplasia; $\mathrm{PIN}$, prostatic intraepithelial neoplasia; $\mathrm{PCa}$, prostate cancer. 
Table 2 Changes of serum CD4, CD8 and CD4/CD8 levels in patients with different pathological types

\begin{tabular}{llll}
\hline Group & CD4 (\%) & CD8 (\%) & CD4/CD8 \\
\hline BPH & $37.14 \pm 5.44$ & $26.67 \pm 6.63$ & $1.5 \mathrm{I} \pm 0.54$ \\
PIN & $34.48 \pm 9.46$ & $23.73 \pm 6.29$ & $1.57 \pm 0.8 \mathrm{I}$ \\
PCa & $28.61 \pm 8.00^{\mathrm{a}, \mathrm{b}}$ & $23.30 \pm 7.83$ & $1.36 \pm 0.60$
\end{tabular}

Notes: ${ }^{a} P=0.000$ vs $B P H ;{ }^{b} P=0.030$ vs PIN. Data are presented as mean \pm standard deviation.

Abbreviations: BPH, benign prostatic hyperplasia; PIN, prostatic intraepithelial neoplasia; PCa, prostate cancer.

$(P=0.000)$ and $\mathrm{PCa}$ and PIN patients $(P=0.030)$. There were no significant differences in the levels of CD8 and CD4/ CD8 between groups $(P>0.05)$.

\section{Differences in S100, CD208 and VEGF expression in biopsy tissues of each group (Table 3 and Figure I)}

The expression level of S100 in each group decreased gradually with the increase of the degree of malignancy. PCa patients showed significantly lower expression of S100 than the other two groups $(P=0.000)$. The expression level of CD208 in each group also decreased gradually with the progression of the disease. The expression of CD208 in PCa patients was the lowest of the three groups, and the difference was statistically significant between PCa and BPH patients $(P=0.000)$. The difference in CD208 expression in PIN and BPH patients was also statistically significant $(P=0.045)$. The expression level of VEGF in each group increased gradually with the increase of the malignant degree of the disease statistical significance, $P=0.000$.

\section{Differences in CD4, CD8 and CD4/CD8 levels in 30 patients with $\mathrm{PCa}$ based on PSA levels and Gleason score (Table 4)}

With the increase in PSA, the expression of peripheral blood CD4 in patients with PCa was gradually reduced. The difference was statistically significant between the two groups $(P=0.000)$. The expression level of CD8 in group B was significantly lower than that in group $\mathrm{A}(P=0.025)$. No significant

Table 3 Expression density changes of SI00, CD208 and VEGF of three groups patients with local tissue biopsy

\begin{tabular}{llll}
\hline Group & S100 & CD208 & VEGF \\
\hline BPH & $26.97 \pm 4.35$ & $2.23 \pm 0.68$ & $60.21 \pm 7.22$ \\
PIN & $21.70 \pm 2.38^{\mathrm{c}}$ & $1.60 \pm 0.37^{\mathrm{e}}$ & $71.75 \pm 4.37^{\mathrm{h}}$ \\
$\mathrm{PCa}$ & $13.20 \pm 5.08^{\mathrm{a}, \mathrm{b}}$ & $1.12 \pm 0.58^{\mathrm{d}}$ & $83.77 \pm 5.48^{\mathrm{fg}}$ \\
\hline
\end{tabular}

Notes: ${ }^{a} P=0.000$ vs $B P H ;{ }^{b P}=0.000$ vs $P I N ;{ }^{c} P=0.015$ vs $B P H ;{ }^{d} P=0.000$ vs $B P H$; ${ }^{e} P=0.045$ vs $B P H ; f P=0.000$ vs $B P H ; s P=0.000$ vs $P I N ;{ }^{~} P=0.000$ vs $B P H$. Data are presented as mean \pm standard deviation.

Abbreviations: VEGF, vascular endothelial growth factor; $\mathrm{BPH}$, benign prostatic hyperplasia; PIN, prostatic intraepithelial neoplasia; PCa, prostate cancer. differences in expression levels of CD8 were seen between group $\mathrm{C}$ and group A or group $\mathrm{B}(>0.05)$. The ratio of CD4/ CD8 decreased with the increase of PSA, and the differences between group $C$ and group $B$ and between group $C$ and group $B$ were statistically significant $(P<0.05)$. There was no significant difference between group $\mathrm{B}$ and group $\mathrm{C}(P>0.05)$.

The expression of CD4 in PCa patients decreased with the increase in Gleason score, and the difference was statistically significant between the two groups $(P=0.000)$. The expression level of CD8 in PCa patients was not statistically significant between groups, $(P>0.05)$, and there was no significant difference in the $\mathrm{CD} 4 / \mathrm{CD} 8$ ratio between the two groups $(P>0.05)$.

\section{Differences in SI00, CD208 and VEGF expression in $\mathrm{PCa}$ patients based on PSA levels and Gleason score (Table 5)}

With increased PSA levels, the expression of S100 in PCa patients decreased significantly. The differences in $\mathrm{S} 100$ expression was significant between all groups $(P<0.05)$. The expression of CD208 decreased with increased PSA levels in PCa patients, and the differences between group B and group $\mathrm{A}$ and between group $\mathrm{C}$ and group $\mathrm{A}$ were statistically significant $(P=0.000)$. However, there was no significant difference in the expression of CD208 between group $\mathrm{C}$ and group B $(P>0.05)$. The expression level of VEGF increased gradually with the increase of PSA, and this difference was statistically significant in both groups $(P<0.05)$.

The expression of S100 in PCa patients decreased significantly with the increase in Gleason score $(P<0.05)$. In addition, the expression level of CD208 in patients with $\mathrm{PCa}$ decreased significantly with the increase in Gleason score $(P<0.05)$. The expression level of VEGF in patients with PCa increased with the increase in Gleason score, and the difference was statistically significant between the two groups $(P<0.05)$.

\section{Correlation between expression of VEGF and S100 and CD208 expression levels in PCa patients}

There was no correlation between VEGF and S100 expression levels $(P=0.839)$ (Figure 2A). However, there was a negative correlation between VEGF and CD208 expression levels $(P=0.001)$ (Figure 2B).

\section{Discussion}

As important antigen-presenting cells (APCs), DCs function at the interface of the innate and adaptive immune systems. 


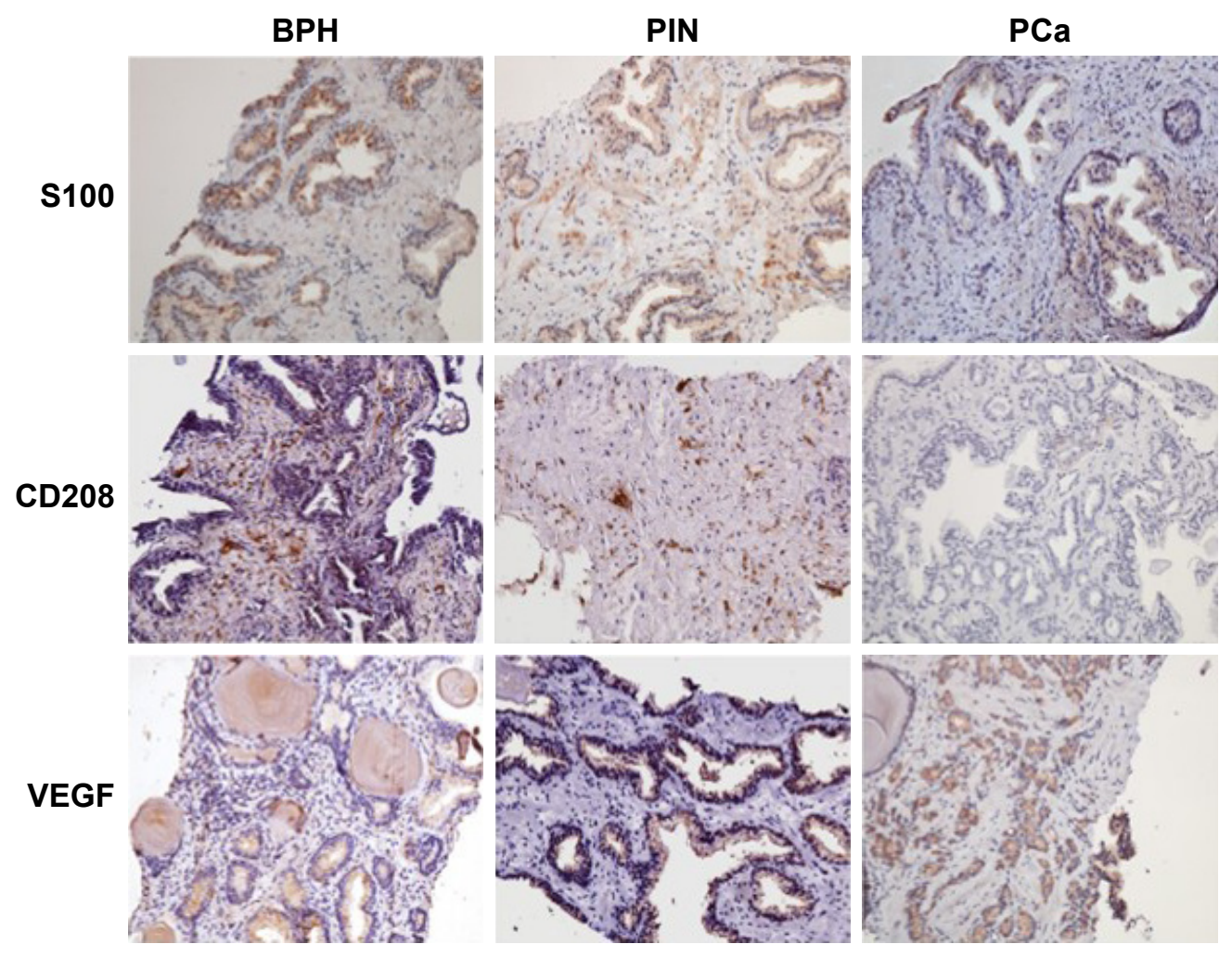

Figure I Immunohistochemistry results of S100, CD208 and VEGF expression in biopsy tissues of each group ( $\times 200$ original magnification).

Abbreviations: PCa, prostate cancer; BPH, benign prostatic hyperplasia; PIN, prostatic intraepithelial neoplasia; VEGF, vascular endothelial growth factor.

In their role as master APCs, DCs induce adaptive responses by processing and presenting antigens on major histocompatibility complex (MHC) molecules to naïve T lymphocytes in lymphoid organs. ${ }^{25} \mathrm{DCs}$ are the most important APC population for regulating innate and adaptive immune responses. They are required for converting naïve resting $T$ cells into potent tumor-reactive cytotoxic helper $\mathrm{CD}^{+} \mathrm{T}$ cells. ${ }^{26} \mathrm{In}$ this study, we showed that $\mathrm{S} 100$, which is expressed by all DCs, and CD208, which is expressed by mature and functional DCs, were lower than in PCa patients than in BPH

Table 4 Differences in CD4, CD8 and CD4/CD8 levels in 30 PCa patients based on PSA levels and different Gleason score

\begin{tabular}{llll}
\hline Group (N) & CD4 (\%) & CD8 (\%) & CD4/CD8 \\
\hline A (I0) & $38.03 \pm 4.10$ & $27.75 \pm 7.11$ & $1.58 \pm 0.45$ \\
B (I0) & $27.72 \pm 2.53^{\mathrm{c}}$ & $19.90 \pm 5.95^{\mathrm{d}}$ & $1.56 \pm 0.73$ \\
C (I0) & $20.09 \pm 1.60^{\mathrm{a}, \mathrm{b}}$ & $23.84 \pm 7.83$ & $0.97 \pm 0.35^{\mathrm{e}, \mathrm{f}}$ \\
D (8) & $38.74 \pm 4.60$ & $27.47 \pm 5.5 \mathrm{I}$ & $1.50 \pm 0.24$ \\
E (9) & $30.42 \pm 2.44^{\mathrm{i}}$ & $20.68 \pm 6.45$ & $1.58 \pm 0.52$ \\
F (I3) & $21.12 \pm 2.57^{\mathrm{g}, \mathrm{h}}$ & $22.46 \pm 8.84$ & $\mathrm{I} .16 \pm 0.72$ \\
\hline
\end{tabular}

Notes: Group A, PSA < $10 \mathrm{ng} / \mathrm{mL}$; group B, PSA $10-20 \mathrm{ng} / \mathrm{mL}$; group C, PSA $>20 \mathrm{ng} / \mathrm{mL}$; group $D$, Gleason grade $<7$; group E, Gleason grade 7; group $F$, Gleason grade $>7$. ${ }^{a} P=0.000$ vs group $A ;{ }^{b} P=0.000$ vs group $B ; c P=0.000$ vs group $A ;{ }^{d} P=0.025$ vs group $A ;{ }^{e} P=0.016$ vs group $A ;{ }^{f} P=0.019$ vs group $B ; s P=$ 0.000 vs group $D ;{ }^{\mathrm{h} P}=0.000$ vs group $E ; \mathrm{P}=0.000$ vs group $D$. $N$ is the numbers of the cases. Data are presented as mean \pm standard deviation.

Abbreviations: $\mathrm{PCa}$, prostate cancer; PSA, prostate-specific antigen. and PIN patients. The infiltration density level of total DCs and mature and functional DCs decreased with the increase of PSA level and Gleason score. Prostate tumor cells cannot only induce the apoptosis of mature DCs but also inhibit the differentiation and maturation of immature DCs, thereby reducing the number of DCs and inhibiting their function. DC deficiency and dysfunction are immune escape mechanisms of $\mathrm{PCa} .{ }^{27} \mathrm{We}$ also found that $\mathrm{CD} 4^{+} \mathrm{T}$ cells in PCa patients

Table 5 Differences in S100, CD208 and VEGF expression in PCa patients based on PSA levels and different Gleason score

\begin{tabular}{llll}
\hline Group & SIO0 (N) & CD208 (N) & VEGF (N) \\
\hline A & $18.77 \pm 4.10(6)$ & $1.90 \pm 0.37(6)$ & $76.60 \pm 3.98(7)$ \\
B & $12.57 \pm 1.91(7)^{\mathrm{c}}$ & $0.97 \pm 0.31(7)^{\mathrm{e}}$ & $83.27 \pm 3.24(9)^{\mathrm{h}}$ \\
C & $9.06 \pm 2.43(9)^{\mathrm{a}, \mathrm{b}}$ & $0.68 \pm 0.10(8)^{\mathrm{d}}$ & $88.38 \pm 3.62(9)^{\mathrm{fg}}$ \\
D & $18.56 \pm 3.81(5)$ & $1.84 \pm 0.57(5)$ & $76.60 \pm 4.16(6)$ \\
E & $14.16 \pm 4.50(8)^{\mathrm{k}}$ & $1.13 \pm 0.42(8)^{\mathrm{n}}$ & $82.40 \pm 4.26(8)^{\mathrm{q}}$ \\
F & $9.33 \pm 2.65(9)^{\mathrm{ij}}$ & $0.68 \pm 0.10(8)^{\mathrm{l}, \mathrm{m}}$ & $87.78 \pm 3.56(1 \mathrm{I})^{\mathrm{o}, \mathrm{p}}$ \\
\hline
\end{tabular}

Notes: Group A, PSA < $10 \mathrm{ng} / \mathrm{mL}$; group B, PSA $10-20 \mathrm{ng} / \mathrm{mL}$; group C, PSA $>20 \mathrm{ng} / \mathrm{mL}$; group D, Gleason grade $<7$; group E, Gleason grade 7; group $F$, Gleason grade $>7$. ${ }^{a} P=0.000$ vs group $A ;{ }^{b} P=0.024$ vs group $B ;{ }^{c} P=0.00$ I vs group $A$; ${ }^{d} P=0.000$ vs group $A ;{ }^{e} P=0.000$ vs group $A ; f P=0.000$ vs group $A ;{ }^{i} P=0.006$ vs group $B ;{ }^{h} P=0.001$ vs group $A ; i P=0.000$ vs group $D ; i P=0.014$ vs group $E ;{ }^{k} P=0.049$ vs group $D ; \mid P=0.000$ vs group $D ;{ }^{m} P=0.029$ vs group $E ;{ }^{n} P=0.004$ vs group $D ; \circ P=$ 0.000 vs group $D ; P P=0.007$ vs group $E ; A P=0.012$ vs group $D$. $N$ is the numbers of the cases. Data are presented as mean \pm standard deviation.

Abbreviations: $\mathrm{PCa}$, prostate cancer; VEGF, vascular endothelial growth factor; PSA, prostate-specific antigen. 

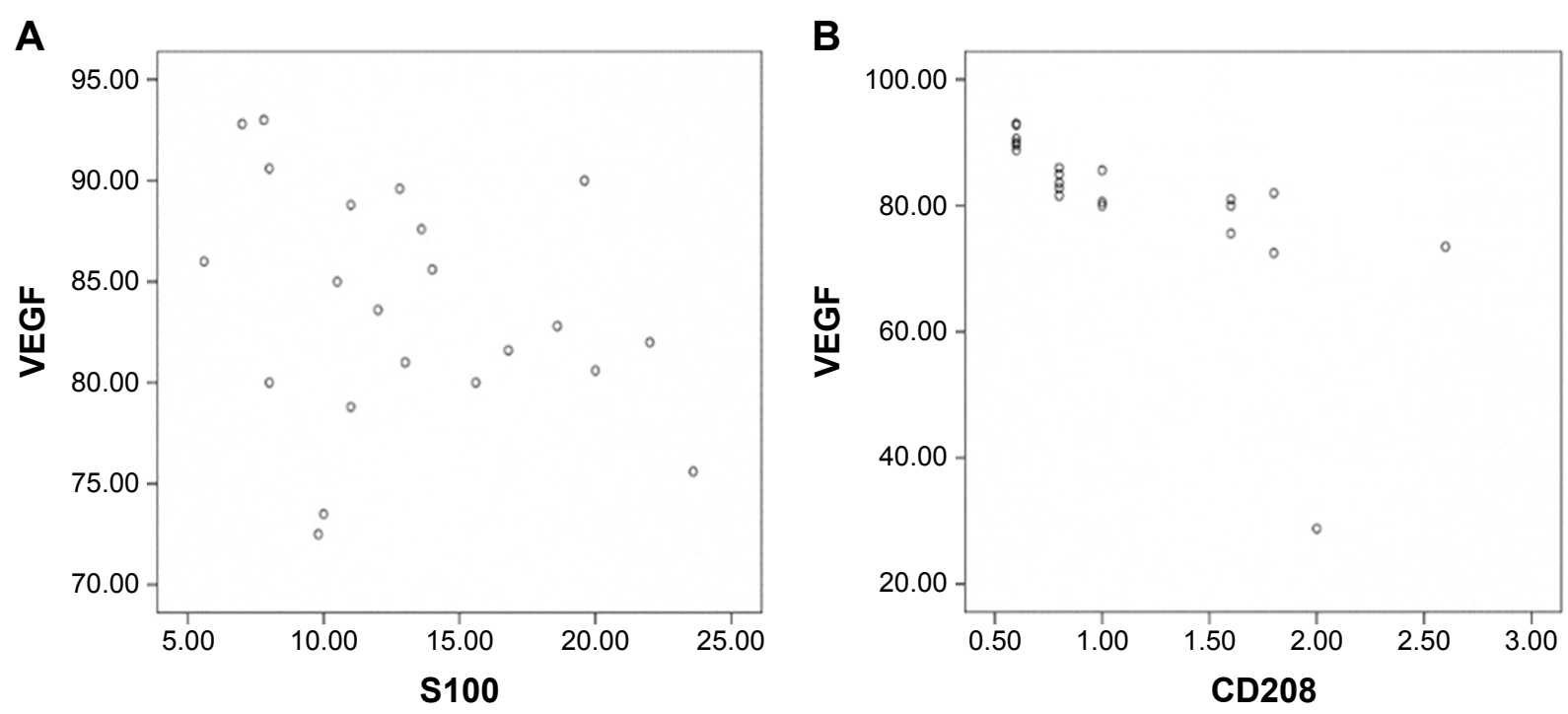

Figure 2 (A) VEGF and SI00 in PCa infiltration density scatter diagram. (B) VEGF and CD208 in PCa infiltration density scatter diagram. Abbreviations: PCa, prostate cancer; VEGF, vascular endothelial growth factor.

were more suppressed than in BPH and PIN patients. The decrease of $\mathrm{CD}^{+}$cells was enhanced with increasing PSA levels and Gleason scores. This indicates that PCa patients have abnormal immune functions and that $\mathrm{DC}$ activation and function were inhibited by the cancer cells. Furthermore, cancer cell-induced suppression of $\mathrm{CD}^{+} \mathrm{T}$-cell numbers leads to the inhibition of immune system functions. This effect was achieved by inhibiting DCs. The number of tumorinfiltrating DCs in tumor patients was reduced and cells had functional defects. Compared with the normal DC phase, tumor-infiltrating DC deficiency stimulates the proliferation of $\mathrm{T}$ cells and the ability to produce cytokines. This is one of the mechanisms of tumor immune escape. ${ }^{8} \mathrm{CD} 4 / \mathrm{CD} 8$ ratio is a key marker for clinical outcome and immune dysfunction, and in the results of this study, the ratio of $\mathrm{CD} 4 / \mathrm{CD} 8$ in $\mathrm{PCa}$ was lower than in $\mathrm{BPH}$, but this difference was not statistically significant. We also got the same result between group $F$ and group D. We thought that the possible reason was that the number of cases was small. Thus, we hope to increase the number of cases in future studies. Furthermore, we did not observe any difference in $\mathrm{CD} 8^{+} \mathrm{T}$ cell levels with the progression of $\mathrm{PCa}$. Although a lot of research has shown that the $\mathrm{CD} 8^{+} \mathrm{T}$ cell is one of the important immune $\mathrm{T}$ cells involved in tumor immunity, it could inhibit the process of cancer, but some other results have shown that the $\mathrm{CD} 8^{+}$ T-cell subpopulation also has potent suppressive activity in prostate cancer, and it could inhibit tumor immunity. ${ }^{28,29}$

VEGF was initially identified due to its capacity to enhance vascular permeability and endothelial cell growth. It is now recognized as the key inducer of angiogenesis in tumors. VEGF is secreted by most tumors and myeloid infiltrates. ${ }^{30} \mathrm{~A}$ high level of VEGF in the serum of cancer patients is correlated with increased metastasis, tumor aggressiveness, and poor prognosis. VEGF suppresses the maturation capacity and the differentiation of DCs and other immune cells from hematopoietic lineages ${ }^{31}$ DCs which have been damaged cannot effectively present tumor antigen to the body's immune system. In the past study, we inhibited VEGF expression and found that it can improve DC and $T$ cells proliferation and also promote activation of anti-tumor immunocytes in the VEGF-inhibited microenvironment. ${ }^{32} \mathrm{In}$ this study, we found that VEGF levels were higher in PCa patients than in PIN and BPH patients. The infiltration density level of VEGF-positive cells increased with increased PSA level and Gleason score. The infiltration density of VEGF-positive cells was negatively correlated with the maturation and function of DCs. VEGF inhibited the number of mature and functional DCs, thereby inhibiting the function of DCs and further inhibiting tumor immunity. VEGF expression had a relationship with the degree of malignancy of prostate cancer. ${ }^{33}$ With the progress of prostate cancer, DC maturation was inhibited, tumor immunity was inhibited too. VEGF might have an influence on the biophysical properties of DC, including electrophoretic mobility, osmotic fragility, viscoelasticity, and transmigration, ${ }^{34}$ so the total number of DCs was reduced in prostate cancer, and the number of total DCs reduced with the progress of prostate cancer. VEGF also acts, through the induction of programmed death ligand 1(PDL1 or B7-H1) on myeloid DCs, ${ }^{35}$ to inhibit the immunity of cancer, but the real mechanism of how VEGF 
inhibits mature DCs still needs to be studied in the future. The therapeutic efficacies may be improved by blocking the signaling pathway of VEGF in an appropriate manner.

\section{Conclusion}

The numbers of total DCs, mature and functional DCs, and $\mathrm{CD}^{+} \mathrm{T}$ cells were inhibited in $\mathrm{PCa}$, and the inhibitory effect was enhanced with increased malignancy. In addition, the infiltration density of VEGF-positive cells was increased in $\mathrm{PCa}$, and this increase was associated with an increased malignant degree of PCa. The inhibition of tumor immunity in patients with $\mathrm{PCa}$ is achieved by inhibiting the function of DCs. VEGF plays an important role in the inhibition of the maturation and function of DCs, and this inhibition is gradually increased with an increasing malignant degree of PCa.

\section{Acknowledgments}

This work was supported by the National Natural Science Foundation of China $(81271597,81401421)$ and capacity building project of clinical auxiliary department (SHDC22015001).

\section{Author contributions}

All authors contributed toward data analysis, drafting and critically revising the paper and agree to be accountable for all aspects of the work. Wen-kun Bai and Wei Zhang are co-first authors.

\section{Disclosure}

The authors report no conflicts of interest in this work.

\section{References}

1. Siegel R, Ma J, Zou Z, Jemal A. Cancer statistics, 2014. CA Cancer J Clin. 2014;64(1):9-29.

2. Simons JW. Prostate cancer immunotherapy: beyond immunity to curability. Cancer Immunol Res. 2014;2(11):1034-1043.

3. Zhang SY, Thara E, Quinn DI, Dorff TB. Blood cells and their use in active immunotherapy of prostate cancer. Hum Vaccin Immunother. 2012;8(4): 528-533.

4. Cintolo JA, Datta J, Mathew SJ, Czerniecki BJ. Dendritic cell-based vaccines: barriers and opportunities. Future Oncol. 2012;8(10): 1273-1299.

5. Whitmore WF, Gittes RF. Studies on the prostate and testis as immunologically privileged sites. Cancer Treat Rep. 1977;61(2):217-222.

6. Drake CG. Prostate cancer as a model for tumour immunotherapy. Nat Rev Immunol. 2010;10(8):580-593.

7. Jahnisch H, Fussel S, Kiessling A, et al. Dendritic cell-based immunotherapy for prostate cancer. Clin Dev Immunol. 2010;2010:517493.

8. Troy A, Davidson P, Atkinson C, Hart D. Phenotypic characterisation of the dendritic cell infiltrate in prostate cancer. J Urol. 1998;160(1): 214-219.

9. Aalamian M, Pirtskhalaishvili G, Nunez A, et al. Human prostate cancer regulates generation and maturation of monocyte-derived dendritic cells. Prostate. 2001;46(1):68-75.
10. Luczynska E, Gasinska A, Blecharz P, Stelmach A, Jereczek-Fossa BA, Reinfuss M. Value of perfusion CT parameters, microvessl density and VEGF expression in differentiation of benign and malignant prostate tumours. Pol J Pathol. 2014;65(3):229-236.

11. Huang Y, Yuan J, Righi E, et al. Vascular normalizing doses of antiangiogenic treatment reprogram the immunosuppressive tumor microenvironment and enhance immunotherapy. Proc Natl Acad Sci U S A. 2012;109(43):17561-17566.

12. Franovic A, Holterman CE, Payette J, Lee S. Human cancers converge at the HIF-2alpha oncogenic axis. Proc Natl Acad Sci U S A. 2009; 106(50):21306-21311.

13. Gallucci S, Lolkema M, Matzinger P. Natural adjuvants: endogenous activators of dendritic cells. Nat Med. 1999;5(11):1249-1255.

14. Ni YH, Wang ZY, Huang XF, et al. Effect of siRNA-mediated downregulation of VEGF in Tca8113 cells on the activity of monocytederived dendritic cells. Oncology Lett. 2012;3(4):885-892.

15. Sugiyama M, Kakeji Y, Tsujitani S, et al. Antagonism of VEGF by genetically engineered dendritic cells is essential to induce antitumor immunity against malignant ascites. Molec Cancer Therapeut. 2011;10(3): 540-549.

16. Rini BI, Weinberg V, Fong L, Conry S, Hershberg RM, Small EJ. Combination immunotherapy with prostatic acid phosphatase pulsed antigen-presenting cells (provenge) plus bevacizumab in patients with serologic progression of prostate cancer after definitive local therapy. Cancer. 2006;107(1):67-74.

17. Xu G, Wu J, Yao MH, et al. Parameters of prostate cancer at contrastenhanced ultrasound: correlation with prostate cancer risk. Int J Clin Exp Med. 2015;8(2):2562-2569.

18. Heidenreich A, Bastian PJ, Bellmunt J, et al. EAU guidelines on prostate cancer. Part 1: screening, diagnosis, and local treatment with curative intent-update 2013. Eur Urol. 2014;65(1):124-137.

19. Roobol MJ, van Vugt HA, Loeb S, et al. Prediction of prostate cancer risk: the role of prostate volume and digital rectal examination in the ERSPC risk calculators. Eur Urol. 2012;61(3):577-583.

20. Roobol MJ, Zhu X, Schroder FH, et al. A calculator for prostate cancer risk 4 years after an initially negative screen: findings from ERSPC Rotterdam. Eur Urol. 2013;63(4):627-633.

21. Poliani PL, Kisand K, Marrella V, et al. Human peripheral lymphoid tissues contain autoimmune regulator-expressing dendritic cells. Am J Pathol. 2010;176(3):1104-1112.

22. Thongtharb A, Uchida K, Chambers JK, Kagawa Y, Nakayama H. Histological and immunohistochemical studies on primary intracrania canine histiocytic sarcomas. J Veterin Med Sci. 2016;78(4):593-599.

23. Bauer K, Michel S, Reuschenbach M, Nelius N, von Knebel Doeberitz M, Kloor M. Dendritic cell and macrophage infiltration in microsatelliteunstable and microsatellite-stable colorectal cancer. Fam Cancer. 2011;10(3):557-565.

24. Duque JL, Loughlin KR, AdamRM, KantoffP, MazzucchiE, Freeman MR. Measurement of plasma levels of vascular endothelial growth factor in prostate cancer patients: relationship with clinical stage, Gleason score, prostate volume, and serum prostate-specific antigen. Clinics (Sao Paulol). 2006;61(5):401-408.

25. Datta J, Terhune JH, Lowenfeld L, et al. Optimizing dendritic cellbased approaches for cancer immunotherapy. Yale J Biol Med. 2014 87(4):491-518.

26. Thara E, DorffTB, Pinski JK, Quinn DI. Vaccine therapy with sipuleucelT (Provenge) for prostate cancer. Maturitas. 2011;69(4):296-303.

27. Pirtskhalaishvili G, Shurin GV, Gambotto A, et al. Transduction of dendritic cells with Bcl- $\mathrm{x}_{\mathrm{L}}$ increases their resistance to prostate cancerinduced apoptosis and antitumor effect in mice. J Immunol. 2000;165(4): 1956-1964.

28. Kiniwa Y, Miyahara Y, Wang HY, et al. $\mathrm{CD}^{+}$Foxp3+ regulatory $\mathrm{T}$ cells mediate immunosuppression in prostate cancer. Clin Cancer Res. 2007;13(23):6947-6958.

29. Yu P, Steel JC, Zhang M, et al. Simultaneous inhibition of two regulatory T-cell subsets enhanced Interleukin-15 efficacy in a prostate tumor model. Proc Natl Acad Sci U S A. 2012;109(16):6187-6192. 
30. Yang L, Carbone DP. Tumor-host immune interactions and dendritic cell dysfunction. Adv Cancer Res. 2004;92:13-27.

31. Sheng KC, Wright MD, Apostolopoulos V. Inflammatory mediators hold the key to dendritic cell suppression and tumor progression. Curr Medicinal Chem. 2011;18(36):5507-5518.

32. Zhang W, Shou WD, Xu YJ, Bai WK, Hu B. Low-frequency ultrasoundinduced VEGF suppression and synergy with dendritic cell-mediated antitumor immunity in murine prostate cancer cells in vitro. Scientific Rep. 2017;7(1):5778

33. Kostis G, Ioannis L, Helen K, Helen P. The expression of vascular endothelial growth factor-C correlates with lymphatic microvessel density and lymph node metastasis in prostate carcinoma: an immunohistochemical study. Urol Ann. 2014;6(3):224-230.
34. Hu ZQ, Xue H, Long JH, et al. Biophysical properties and motility of human mature dendritic cells deteriorated by vascular endothelial growth factor through cytoskeleton remodeling. Int J Molec Sci. 2016; 17(11):1756.

35. Curiel TJ, Wei S, Dong H, et al. Blockade of B7-H1 improves myeloid dendritic cell-mediated antitumor immunity. Nat Med. 2003;9(5): $562-567$.

\section{Publish your work in this journal}

OncoTargets and Therapy is an international, peer-reviewed, open access journal focusing on the pathological basis of all cancers, potential targets for therapy and treatment protocols employed to improve the management of cancer patients. The journal also focuses on the impact of management programs and new therapeutic agents and protocols on

\section{Dovepress}

patient perspectives such as quality of life, adherence and satisfaction. The manuscript management system is completely online and includes a very quick and fair peer-review system, which is all easy to use. Visit http://www.dovepress.com/testimonials.php to read real quotes from published authors. 